\title{
Asymptotic enumeration of sparse uniform linear hypergraphs with given degrees
}

\author{
Vladimir Blinovsky \\ Instituto de Matemática e Estatística \\ Universidade de São Paulo, 05508-090, Brazil \\ Institute for Information Transmission Problems \\ Russian Academy of Sciences, Moscow 127994, Russia \\ vblinovs@yandex.ru \\ Catherine Greenhill * \\ School of Mathematics and Statistics \\ UNSW Australia \\ Sydney NSW 2052, Australia \\ c.greenhill@unsw.edu.au
}

Submitted: Aug 25, 2015; Accepted: Jul 20, 2016; Published: Aug 5, 2016

Mathematics Subject Classifications: 05C30, 05C65

\begin{abstract}
A hypergraph is simple if it has no loops and no repeated edges, and a hypergraph is linear if it is simple and each pair of edges intersects in at most one vertex. For $n \geqslant 3$, let $r=r(n) \geqslant 3$ be an integer and let $\boldsymbol{k}=\left(k_{1}, \ldots, k_{n}\right)$ be a vector of nonnegative integers, where each $k_{j}=k_{j}(n)$ may depend on $n$. Let $M=M(n)=$ $\sum_{j=1}^{n} k_{j}$ for all $n \geqslant 3$, and define the set $\mathcal{I}=\{n \geqslant 3 \mid r(n)$ divides $M(n)\}$. We assume that $\mathcal{I}$ is infinite, and perform asymptotics as $n$ tends to infinity along $\mathcal{I}$. Our main result is an asymptotic enumeration formula for linear $r$-uniform hypergraphs with degree sequence $\boldsymbol{k}$. This formula holds whenever the maximum degree $k_{\max }$ satisfies $r^{4} k_{\max }^{4}\left(k_{\max }+r\right)=o(M)$. Our approach is to work with the incidence matrix of a hypergraph, interpreted as the biadjacency matrix of a bipartite graph, enabling us to apply known enumeration results for bipartite graphs. This approach also leads to a new asymptotic enumeration formula for simple uniform hypergraphs with specified degrees, and a result regarding the girth of random bipartite graphs with specified degrees.
\end{abstract}

Keywords: hypergraphs; linear hypergraphs; asymptotic enumeration; switchings

* Research supported by the Australian Research Council Discovery Project DP140101519. 


\section{Introduction}

Hypergraphs are combinatorial structures which can model very general relational systems, including some real-world networks $[6,8,11]$. Formally, a hypergraph or set system is defined as a pair $(V, E)$, where $V$ is a finite set and $E$ is a multiset of multisubsets of $V$. (We refer to elements of $E$ as edges.) Note that under this definition, a hypergraph may contain repeated edges and an edge may contain repeated vertices.

Any 2-element multisubset of an edge $e \in E$ is called a link in $e$. If a vertex $v$ has multiplicity at least 2 in the edge $e$, we say that $v$ is a loop in $e$. (So every loop in $e$ is also a link in e.) The multiplicity of a link $\{x, y\}$ is the number of edges in $E$ which contain $\{x, y\}$ (counting multiplicities). A double link is a link with multiplicity 2.

A hypergraph is simple if it has no loops and no repeated edges: that is, $E$ is a set of edges, and each edge is a subset of $V$. Here it is possible that distinct edges may have more than one vertex in common. (This definition of simple hypergraph appears to be standard, and matches the definition of simple hypergraphs given by Berge [1] in the case of uniform hypergraphs.) A hypergraph is called linear if it has no loops and each pair of distinct edges intersect in at most one vertex. (Note that linear hypergraphs are also simple, when $r \geqslant 2$.) Linear hypergraphs have been well-studied in many contexts (sometimes they have been referred to as "simple hypergraphs"). See for example [3, 7, 12, 19].

For a positive integer $r$, the hypergraph $(V, E)$ is $r$-uniform if each edge $e \in E$ contains exactly $r$ vertices (counting multiplicities). Uniform hypergraphs are a particular focus of study, not least because a 2-uniform hypergraph is precisely a graph. We seek an asymptotic enumeration formula for the number of linear $r$-uniform hypergraphs with a given degree sequence, when the maximum degree is not too large (the sparse range), and allowing $r$ to grow slowly with $n$.

To state our result precisely, we need some definitions. Write $[a]=\{1,2, \ldots, a\}$ for all positive integers $a$. Given nonnegative integers $a, b$, let $(a)_{b}$ denote the falling factorial $a(a-1) \cdots(a-b+1)$. We are given a degree sequence $\boldsymbol{k}=\boldsymbol{k}(n)=\left(k_{1}, \ldots, k_{n}\right)$ with sum $M=M(n)=\sum_{i=1}^{n} k_{i}$, and we are also given an integer $r=r(n) \geqslant 3$, for each $n \geqslant 3$. Let $k_{\max }=k_{\max }(n)=\max _{j=1}^{n} k_{j}$ for all $n \geqslant 3$. For each positive integer $t$, define

$$
M_{t}=M_{t}(n)=\sum_{i=1}^{n}\left(k_{i}\right)_{t} .
$$

Then $M_{1}=M$ and $M_{t} \leqslant k_{\max } M_{t-1}$ for $t \geqslant 2$.

Let $\mathcal{H}_{r}(\boldsymbol{k})$ denote the set of simple $r$-uniform hypergraphs on the vertex set $[n]$ with degree sequence given by $\boldsymbol{k}=\left(k_{1}, \ldots, k_{n}\right)$, and let $\mathcal{L}_{r}(\boldsymbol{k})$ be the set of all linear hypergraphs in $\mathcal{H}_{r}(\boldsymbol{k})$. Note that $\mathcal{H}_{r}(\boldsymbol{k})$ and $\mathcal{L}_{r}(\boldsymbol{k})$ are both empty unless $r$ divides $M$. Our main theorem is the following.

Theorem 1.1. For $n \geqslant 3$, let $r=r(n) \geqslant 3$ be an integer and let $\boldsymbol{k}=\left(k_{1}, \ldots, k_{n}\right)$ be a vector of nonnegative integers, where each $k_{j}=k_{j}(n)$ may depend on $n$. Let $k_{\max }=$ $k_{\max }(n)=\max _{j=1}^{n} k_{j}$ for all $n \geqslant 3$. Define $M=M(n)=\sum_{j=1}^{n} k_{j}$ for all $n \geqslant 3$, and suppose that the set

$$
\mathcal{I}=\{n \geqslant 3 \mid r(n) \text { divides } M(n)\}
$$


is infinite. Suppose that $M \rightarrow \infty$ and $r^{4} k_{\max }^{4}\left(k_{\max }+r\right)=o(M)$ as $n$ tends to infinity along elements of $\mathcal{I}$. Then

$$
\begin{aligned}
& \left|\mathcal{L}_{r}(\boldsymbol{k})\right| \\
& =\frac{M !}{(M / r) !(r !)^{M / r} \prod_{i=1}^{n} k_{i} !} \exp \left(-\frac{(r-1) M_{2}}{2 M}-\frac{(r-1)^{2} M_{2}^{2}}{4 M^{2}}+O\left(\frac{r^{4} k_{\max }^{4}\left(k_{\max }+r\right)}{M}\right)\right) .
\end{aligned}
$$

We believe that Theorem 1.1 is the first asymptotic enumeration by degree sequence for $r$-uniform linear hypergraphs with $r \geqslant 3$, and the first asymptotic enumeration result for sparse hypergraphs which allows $r$ to grow with $n$. The two (non-error) terms within the exponential arise naturally: the first corresponds to the expected number of loops and the second corresponds to the expected number of double links.

A brief survey of the relevant literature is given in the next subsection. Note that when $r=2$ (graphs), our result is weaker than the formula given by McKay and Wormald [17], as their expression has smaller error term and more significant terms. In order to improve the accuracy of Theorem 1.1 to a similar level, a more detailed analysis of double links is required. We will present such an analysis in a future paper.

To obtain Theorem 1.1, we treat the incidence matrix of a hypergraph as the biadjacency matrix of a bipartite graph, thereby enabling us to make use of prior enumeration results for bipartite graphs in order to enumerate linear hypergraphs. In Section 2 we show that some undesirable substructures are rare in random bipartite graphs with the appropriate degrees. As a corollary of this, we obtain a new enumeration result for sparse simple uniform hypergraphs. Theorem 1.1 then follows from a switching argument for bipartite graphs which is used to remove 4-cycles, as these correspond to double links in the hypergraph. This switching argument is presented in Section 3, leading to the proof of Theorem 1.1. Finally in Corollary 3.5 we state a consequence of our calculations relating to the girth of bipartite graphs with specified degrees.

Using this approach of translating the problem to one involving bipartite graphs, it should be possible to relax the uniformity condition, perhaps by allowing the number of edges with a given size to be specified up to a maximum edge size (which may grow modestly with $n$ ). Such a generalisation has not been performed here.

\subsection{History}

In the case of graphs, the best asymptotic formula in the sparse range is given by McKay and Wormald [17]. See that paper for further history of the problem. The dense range was treated in $[15,16]$, but there is a gap between these two ranges in which nothing is known.

An early result in the asymptotic enumeration of hypergraphs was given by Cooper et al. [4], who considered simple $k$-regular hypergraphs when $k=O(1)$. More recently, Dudek et al. [5] proved an asymptotic formula for simple $k$-regular hypergraphs with $k=o\left(n^{1 / 2}\right)$. In [2] this was extended to irregular sequences, with an improved error bound. We restate this result below. 
Theorem 1.2. [2, Theorem 1.1] Let $r \geqslant 3$ be a fixed integer. Let $\boldsymbol{k}, M$ and $k_{\max }$ be defined as in Theorem 1.1. Assume that $r$ divides $M$ for infinitely many values of $n$. Suppose that $M \rightarrow \infty, k_{\max } \geqslant 2$ and $k_{\max }^{3}=o(M)$ as $n$ tends to infinity along these values. Then

$$
\left|\mathcal{H}_{r}(\boldsymbol{k})\right|=\frac{M !}{(M / r) !(r !)^{M / r} \prod_{i=1}^{n} k_{i} !} \exp \left(-\frac{(r-1) M_{2}}{2 M}+O\left(k_{\max }^{3} / M\right)\right) .
$$

Kuperberg, Lovett and Peled [13] gave an asymptotic formula for the number of dense simple $r$-uniform hypergraphs with a given degree sequence.

\section{Hypergraphs, incidence matrices and bipartite graphs}

Suppose that $G$ is an $r$-uniform hypergraph with degree sequence $\boldsymbol{k}$ which has no loops (but may have repeated edges). Let $A$ be the $n \times(M / r)$ incidence matrix of $G$, where the rows of the incidence matrix correspond to vertices $1,2, \ldots n$ in that order, and the columns correspond to the edges of the hypergraph, in some order. Then $A$ is a $0-1$ matrix (as $G$ has no loops), the row sums of $A$ are given by $\boldsymbol{k}$ and each column sum of $A$ equals $r$.

If $G$ is simple (that is, if $G \in \mathcal{H}_{r}(\boldsymbol{k})$ ) then all columns of $A$ are distinct, and hence there are precisely $(M / r)$ ! possible (distinct) incidence matrices corresponding to $G$. Conversely, every 0-1 matrix with rows sums given by $\boldsymbol{k}$, column sums all equal to $r$ and with no repeated columns can be interpreted as the incidence matrix of a hypergraph in $\mathcal{H}_{r}(\boldsymbol{k})$.

It will be convenient to work with the bipartite graphs whose biadjacency matrices are the incidence matrices of hypergraphs. Let $\mathcal{B}_{r}(\boldsymbol{k})$ be the set of bipartite graphs with vertex bipartition $\left\{v_{1}, \ldots, v_{n}\right\} \cup\left\{e_{1}, e_{2}, \ldots, e_{M / r}\right\}$, such that degree sequence of $\left(v_{1}, \ldots, v_{n}\right)$ is $\boldsymbol{k}$ and every vertex $e_{j}$ has degree $r$. We sometimes say that a vertex $v_{j}$ is "on the left" and that a vertex $e_{i}$ is "on the right". An example of a 3-uniform hypergraph, its incidence matrix (with edges ordered in lexicographical order) and corresponding bipartite graph is shown in Figure 1.

Double links will be of particular interest: there are two double links in the hypergraph in Figure 1, and each corresponds to a subgraph of the bipartite graph which is isomorphic to $K_{2,2}$. (One is induced by $\left\{v_{1}, v_{2}, e_{1}, e_{2}\right\}$ and the other by $\left\{v_{5}, v_{6}, e_{3}, e_{4}\right\}$.)

It follows from [10, Theorem 1.3] that

$$
\left|\mathcal{B}_{r}(\boldsymbol{k})\right|=\frac{M !}{(r !)^{M / r} \prod_{j=1}^{n} k_{j} !} \exp \left(-\frac{(r-1) M_{2}}{2 M}+O\left(r^{2} k_{\max }^{2} / M\right)\right)
$$

whenever $1 \leqslant r k_{\max }=o\left(M^{1 / 2}\right)$. (In fact, the result of [10] is more accurate but we are unable to exploit the extra accuracy here, so we state a simplified version.)

Next, let $\mathcal{B}_{r}^{(0)}(\boldsymbol{k})$ denote the set of all bipartite graphs in $\mathcal{B}_{r}(\boldsymbol{k})$ such that no two vertices $e_{i_{1}}, e_{i_{2}}$ (on the right) have the same neighbourhood. These bipartite graphs correspond to 0-1 matrices with no repeated columns, which in turn can be viewed as incidence matrices of (simple) hypergraphs in $\mathcal{H}_{r}(\boldsymbol{k})$. Hence

$$
(M / r) !\left|\mathcal{H}_{r}(\boldsymbol{k})\right|=\left|\mathcal{B}_{r}^{(0)}(\boldsymbol{k})\right| .
$$




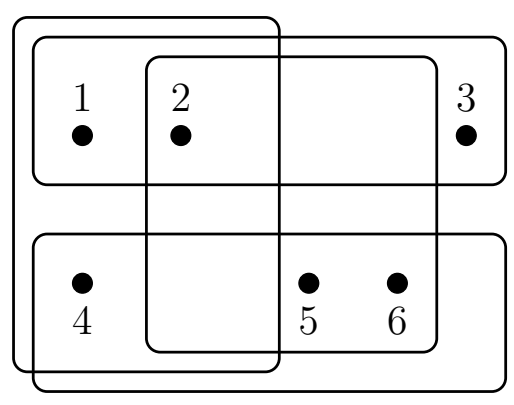

$$
\left(\begin{array}{llll}
1 & 1 & 0 & 0 \\
1 & 1 & 1 & 0 \\
1 & 0 & 0 & 0 \\
0 & 1 & 0 & 1 \\
0 & 0 & 1 & 1 \\
0 & 0 & 1 & 1
\end{array}\right)
$$

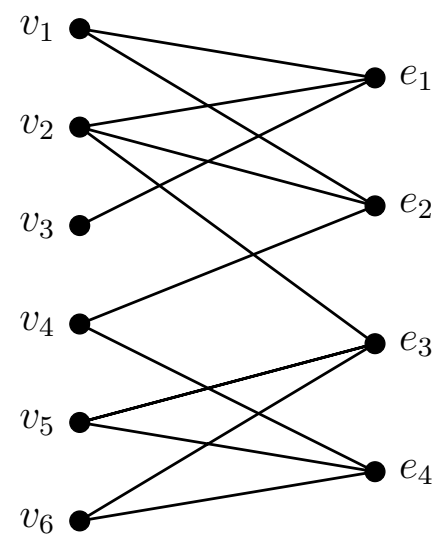

Figure 1: A hypergraph, its incidence matrix and corresponding bipartite graph.

To work towards linear hypergraphs, we identify some desirable properties of the corresponding bipartite graphs. Given an ordered pair of positive integers $(a, b)$, say that the bipartite graph $B$ has a copy of $K_{a, b}$ if $B$ contains a set of $a$ vertices on the left and $b$ vertices on the right which induce a subgraph isomorphic to $K_{a, b}$. This definition is slightly non-standard, since it is not symmetric with respect to $a$ and $b$. However, we will mostly be interested in copies of $K_{2,2}$, in which case there is no asymmetry. We will write " $B$ contains a 4-cycle" rather than " $B$ contains a copy of $K_{2,2}$ ".

Define

$$
N_{2}=3 \max \left\{\lceil\log M\rceil,\left\lceil 2(r-1)^{2} M_{2}^{2} / M^{2}\right\rceil\right\}
$$

and let $\mathcal{B}_{r}^{+}(\boldsymbol{k})$ denote the set of all bipartite graphs $B \in \mathcal{B}_{r}(\boldsymbol{k})$ which satisfy the following properties:

(i) $B$ has no copy of $K_{3,2}$ (with three vertices on the left and two on the right).

(ii) $B$ has no copy of $K_{2,3}$ (with two vertices on the left and three on the right).

(iii) No two 4-cycles in $B$ have a vertex $e_{j}$ (on the right) in common. (This implies that any 4 -cycles in $B$ are edge-disjoint.)

(iv) Any three distinct 4-cycles in $B$ involve at least five vertices on the left. (Together with (iii), this implies that if two distinct 4-cycles share a vertex on the left then any other 4-cycle in $B$ must be vertex-disjoint from the first two.)

(v) The number of 4 -cycles in $B$ is at most $N_{2}$.

To motivate this definition, note that $B \in \mathcal{B}_{r}^{+}(\boldsymbol{k})$ if and only if the corresponding hypergraph $G=G(B)$ satisfies the following properties:

(i) The intersection of any two edges of $G$ contains at most two vertices. 
(ii)' Any link has multiplicity at most two in $G$. (That is, the intersection of any three edges of $G$ contains at most one vertex.)

(iii)' No edge of $G$ contains more than one double link. (That is, if $e_{1}$ and $e_{2}$ are edges of $G$ which share a double link then $e_{1}$ is not involved in any other double link in $G$, and similarly for $e_{2}$.)

(iv)' No vertex can belong to three double links, and if a vertex $v$ belongs to two double links (say $\{v, x\}$ and $\{v, y\}$ are both double links) then both $x$ and $y$ belong to precisely one double link.

$(\mathrm{v})^{\prime}$ There are at most $N_{2}$ double links in $G$.

In particular, as $r \geqslant 3$, any hypergraph $G=G(B)$ with $B \in \mathcal{B}_{r}^{+}(\boldsymbol{k})$ has (no loops and) no repeated edges, so is simple.

McKay [14] proved asymptotic formulae for the probability that a randomly chosen bipartite graph with specified degrees contains a fixed subgraph, under certain conditions. We state one of these results below, which will be use repeatedly. (In fact the statement below is a special case of [14, Theorem 3.5(a)], obtained by taking $J=L$ and $H=\varnothing$ in the notation of [14], and with slightly simplified notation.)

Lemma 2.1. ([14, Theorem 3.5(a)]) Let $\mathcal{B}(\boldsymbol{g})$ denote the set of bipartite graphs with vertex bipartition given by $\left\{a_{1}, \ldots, a_{n}\right\} \cup\left\{b_{1}, \ldots, b_{m}\right\}$ and degree sequence

$$
\boldsymbol{g}=\left(g_{1}, \ldots, g_{n} ; g_{1}^{\prime}, \ldots, g_{m}^{\prime}\right) \text {. }
$$

(Here vertex $a_{i}$ has degree $g_{i}$ for $i=1, \ldots, n$, and vertex $b_{j}$ has degree $g_{j}^{\prime}$ for $j=1, \ldots, m$.) Let $L$ be a subgraph of the complete bipartite graph on this vertex bipartition, and let $\mathcal{B}(\boldsymbol{g}, L)$ be the set of bipartite graphs in $\mathcal{B}(\boldsymbol{g})$ which contain $L$ as a subgraph. Write $E_{\boldsymbol{g}}=\sum_{i=1}^{n} g_{i}$ and $E_{\boldsymbol{\ell}}=\sum_{i=1}^{n} \ell_{i}$, where $\boldsymbol{\ell}=\left(\ell_{1}, \ldots, \ell_{n} ; \ell_{1}^{\prime}, \ldots, \ell_{m}^{\prime}\right)$ is the degree sequence of $L$. Finally, let $g_{\max }$ and $\ell_{\max }$ denote the maximum degree in $\boldsymbol{g}$ and $\boldsymbol{\ell}$, respectively, and define

$$
\Gamma=2 g_{\max }\left(g_{\max }+\ell_{\max }-1\right)+2 .
$$

If $E_{\boldsymbol{g}}-\Gamma \geqslant E_{\boldsymbol{\ell}}$ then

$$
\frac{|\mathcal{B}(\boldsymbol{g}, L)|}{|\mathcal{B}(\boldsymbol{g})|} \leqslant \frac{\prod_{i=1}^{n}\left(g_{i}\right)_{\ell_{i}} \prod_{j=1}^{m}\left(g_{j}^{\prime}\right)_{\ell_{j}^{\prime}}}{\left(E_{\boldsymbol{g}}-\Gamma\right)_{E_{\ell}}}
$$

Using this lemma, we now analyse the probability that a uniformly random element of $\mathcal{B}_{r}(\boldsymbol{k})$ satisfies properties (i)-(v).

Theorem 2.2. Under the conditions of Theorem 1.1,

$$
\frac{\left|\mathcal{B}_{r}^{+}(\boldsymbol{k})\right|}{\left|\mathcal{B}_{r}(\boldsymbol{k})\right|}=1+O\left(r^{5} k_{\max }^{4} / M\right) \text {. }
$$


Proof. Throughout this proof, consider a uniformly random element $B \in \mathcal{B}_{r}(\boldsymbol{k})$. We will apply Lemma 2.1 several times with $\boldsymbol{g}=\left(k_{1}, \ldots, k_{n} ; r, \ldots, r\right)$. In each application, $L$ is a subgraph with constant maximum degree. Hence $g_{\max }=\max \left\{k_{\max }, r\right\}$ and

$$
\Gamma=2 g_{\max }\left(g_{\max }+\ell_{\max }-1\right)+2=O\left(r^{2}+k_{\max }^{2}\right) .
$$

For (i), let $v_{j_{1}}, v_{j_{2}}, v_{j_{3}} \in[n]$ be distinct vertices on the left, and let $e_{i_{1}}, e_{i_{2}}$ be distinct vertices on the right. Applying Lemma 2.1 with $L=K_{3,2}$, we find that the probability that $B$ has a copy of $K_{3,2}$ on the vertices $\left\{v_{j_{1}}, v_{j_{2}}, v_{j_{3}}\right\} \cup\left\{e_{i_{1}}, e_{i_{1}}\right\}$ is at most

$$
\frac{r^{2}(r-1)^{2}(r-2)^{2}}{\left(M+O\left(r^{2}+k_{\max }^{2}\right)\right)_{6}}\left(k_{j_{1}}\right)_{2}\left(k_{j_{2}}\right)_{2}\left(k_{j_{3}}\right)_{2} \text {. }
$$

By assumption, $k_{\max }^{2}+r^{2}=o(M)$. Multiplying this by the number of choices for $\left\{e_{i_{1}}, e_{i_{2}}\right\}$ and summing over all choices of $\left(j_{1}, j_{2}, j_{3}\right)$ with $1 \leqslant j_{1}<j_{2}<j_{3} \leqslant n$ shows that the expected number of copies of $K_{3,2}$ in $B$ is at most

$$
\begin{aligned}
\left(\begin{array}{c}
M / r \\
2
\end{array}\right) \sum_{j_{1}<j_{2}<j_{3}}\left(k_{j_{1}}\right)_{2}\left(k_{j_{2}}\right)_{2}\left(k_{j_{3}}\right)_{2} O\left(\frac{r^{6}}{M^{6}}\right) & =O\left(\frac{r^{4} M_{2}^{3}}{M^{4}}\right) \\
& =O\left(r^{4} k_{\max }^{3} / M\right) .
\end{aligned}
$$

Hence property (i) fails with probability $O\left(r^{4} k_{\max }^{3} / M\right)$. For future reference, we note that the argument leading to (2.3) still holds under the weaker condition $r^{4} k_{\max }^{3}=o(M)$ (as this condition still implies that $k_{\max }^{2}+r^{2}=o(M)$, and all other calculations are unchanged).

Repeating this argument with $L=K_{2,3}$ shows that property (ii) fails with probability $O\left(r^{3} k_{\max }^{4} / M\right)$. Using the subgraphs $L$ shown in Figure 2 (a) and (b) we can establish that property (iii) fails with probability $O\left(r^{5} k_{\max }^{4} / M\right)$. Assuming that properties (i)-(iii)

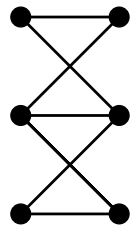

(a)

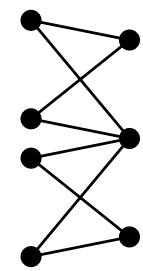

(b)

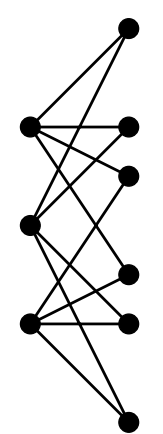

(c)

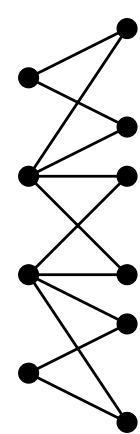

(d)

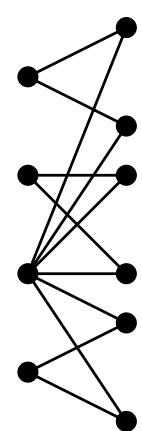

(e)

Figure 2: Rare subgraphs

hold, we can prove that property (iv) holds with probability $O\left(r^{2} k_{\max }^{3} / M\right)$ by considering the subgraphs $L$ shown in Figure 2 (c), (d) and (e). 
Now we turn to $(\mathrm{v})$. Let $Q_{1}=\max \left\{\lceil\log M\rceil,\left\lceil 2(r-1)^{2} M_{2}^{2} / M^{2}\right\rceil\right\}$ and define $d=$ $Q_{1}+1$. We first show that the expected number of sets of $d$ vertex-disjoint 4-cycles in $B$ is $O(1 / M)$. Fix $\left(j_{1}, \ldots, j_{2 d}\right) \in[n]^{2 d}$ such that $k_{j_{\ell}} \geqslant 2$ for $\ell=1,2, \ldots, 2 d$ and $j_{2 \ell-1} \neq j_{2 \ell}$ for $\ell=1,2, \ldots, d$. Let $\left(i_{1}, \ldots, i_{2 d}\right) \in\{1, \ldots, M / r\}^{2 d}$ be a (2d)-tuple of (distinct) edge labels. The probability that there is a 4-cycle on $\left\{v_{j_{2 \ell-1}}, v_{j_{2 \ell}}\right\} \cup\left\{e_{i_{2 \ell-1}}, e_{i_{2 \ell}}\right\}$ for $\ell=1, \ldots, d$ is

$$
\prod_{\ell=1}^{2 d}\left(k_{j_{\ell}}\right)_{2} O\left(\frac{(r(r-1))^{2 d}}{M^{4 d}}\right),
$$

by Lemma 2.1. There are at most $(M / r)^{2 d}$ choices for $\left(i_{1}, \ldots, i_{2 d}\right)$, and for an upper bound we can sum over all possible values of $\left(j_{1}, \ldots, j_{2 d}\right)$. This counts each set of $d$ vertex-disjoint 4 -cycles precisely $4^{d} d$ ! times. It follows that the expected number of sets of $d$ vertex-disjoint 4-cycles in $B$ is

$$
\begin{aligned}
\sum_{\left(j_{1}, \ldots, j_{2 d}\right) \in[n]^{2 d}} \prod_{\ell=1}^{2 d}\left(k_{j_{\ell}}\right)_{2} O\left(\frac{(r-1)^{2 d}}{4^{d} d ! M^{2 d}}\right) & =O\left(\frac{1}{d !}\left(\frac{(r-1)^{2} M_{2}^{2}}{4 M^{2}}\right)^{d}\right) \\
& =O\left(\left(\frac{e(r-1)^{2} M_{2}^{2}}{4 d M^{2}}\right)^{d}\right) \\
& =O\left((e / 8)^{d}\right) \\
& =O(1 / M)
\end{aligned}
$$

by choice of $d$. Next, let $Q_{2}=\max \left\{\lceil\log M\rceil,\left\lceil(r-1)^{4} M_{2}^{2} M_{4} / M^{4}\right\rceil\right\}$ and define $b=Q_{2}+1$. Assuming that properties (iii) and (iv) hold, any 4-cycle in $B$ is either vertex-disjoint from all other 4-cycles in $B$, or shares one vertex on the left with precisely one other 4-cycle in $B$. In the latter case, call such a pair of 4-cycles a fused pair. Arguing as above, the expected number of sets of $b$ fused pairs is at most $O(1 / M)$, by choice of $b$. It follows that with probability $1+O\left(r^{5} k_{\max }^{4} / M\right)$, the number of 4 -cycles in $B$ is at most $Q_{1}+2 Q_{2} \leqslant 3 Q_{1}=N_{2}$, completing the proof.

As a by-product of Theorem 2.2, we obtain a new asymptotic enumeration formula for sparse simple uniform hypergraphs with given degrees, generalising [2, Theorem 1.1] (restated earlier as Theorem 1.2, for ease of comparison): the new formula allows $r$ to grow slowly with $n$, whereas Theorem 1.2 is only valid for fixed $r \geqslant 3$. (The two results match when $r$ is constant.)

Corollary 2.3. For $n \geqslant 3$, let $r=r(n) \geqslant 3$ be an integer and let $\boldsymbol{k}=\boldsymbol{k}(n)=\left(k_{1}, \ldots, k_{n}\right)$ be a vector of nonnegative integers, where each $k_{j}=k_{j}(n)$ may depend on $n$. Let $M=$ $M(n)=\sum_{j=1}^{n} k_{j}$ for all $n \geqslant 3$, and suppose that the set

$$
\mathcal{I}=\{n \geqslant 3 \mid r(n) \text { divides } M(n)\}
$$

is infinite. Suppose that $M \rightarrow \infty$ and $r^{4} k_{\max }^{3}=o(M)$ as $n$ tends to infinity along elements of $\mathcal{I}$. Then

$$
\left|\mathcal{H}_{r}(\boldsymbol{k})\right|=\frac{M !}{(M / r) !(r !)^{M / r} \prod_{i=1}^{n} k_{i} !} \exp \left(-\frac{(r-1) M_{2}}{2 M}+O\left(r^{4} k_{\max }^{3} / M\right)\right)
$$


Proof. As noted earlier, the argument leading to (2.3) is still valid when $r^{4} k_{\max }^{3}=o(M)$. Since $r \geqslant 3$, it follows from $(2.3)$ that $\left|\mathcal{B}_{r}^{(0)}(\boldsymbol{k})\right| /\left|\mathcal{B}_{r}(\boldsymbol{k})\right|=1+O\left(r^{4} k_{\max }^{3} / M\right)$. Combining this with (2.1) and (2.2) completes the proof.

\section{Double links}

For nonnegative integers $d$, let $\mathcal{C}_{d}$ be the set of bipartite graphs in $\mathcal{B}_{r}^{+}(\boldsymbol{k})$ which contain precisely $d 4$-cycles. (The corresponding hypergraph has exactly $d$ double links.) The sets $\mathcal{C}_{d}$ partition $\mathcal{B}_{r}^{+}(\boldsymbol{k})$, and so

$$
\left|\mathcal{B}_{r}^{+}(\boldsymbol{k})\right|=\sum_{d=0}^{N_{2}}\left|\mathcal{C}_{d}\right| .
$$

We estimate this sum using a switching operation which we now define.

An 8-tuple of distinct vertices $T=\left(u_{1}, u_{2}, w_{1}, w_{2}, f_{1}, f_{2}, g_{1}, g_{2}\right)$ is called suitable if

$$
u_{1}, u_{2}, w_{1}, w_{2} \in\left\{v_{1}, \ldots, v_{n}\right\} \text { and } f_{1}, f_{2}, g_{1}, g_{2} \in\left\{e_{1}, \ldots, e_{M / r}\right\} \text {. }
$$

A $d$-switching from $B \in \mathcal{C}_{d}$ is described by a suitable 8-tuple $T$ of vertices of $B$ such that

- $B$ has a 4-cycle on $\left\{u_{1}, u_{2}\right\} \cup\left\{f_{1}, f_{2}\right\}$,

- $w_{1} g_{1}$ and $w_{2} g_{2}$ are edges in $B$.

The corresponding d-switching produces a new bipartite graph $B^{\prime}$ with the same vertex set as $B$ and with edge set

$$
E\left(B^{\prime}\right)=\left(E(B) \backslash\left\{u_{1} f_{1}, u_{2} f_{2}, w_{1} g_{1}, w_{2} g_{2}\right\}\right) \cup\left\{u_{1} g_{1}, u_{2} g_{2}, w_{1} f_{1}, w_{2} f_{2}\right\} .
$$

The d-switching operation is illustrated in Figure 3 below. (Note that in the hypergraph setting, the d-switching replaces the four edges $f_{1}, f_{2}, g_{1}, g_{2}$ of the original hypergraph with the edges $f_{1}^{\prime}, f_{2}^{\prime}, g_{1}^{\prime}, g_{2}^{\prime}$ defined by

$$
f_{j}^{\prime}=\left(f_{j} \backslash\left\{u_{j}\right\}\right) \cup\left\{w_{j}\right\}, \quad g_{j}^{\prime}=\left(g_{j} \backslash\left\{w_{j}\right\}\right) \cup\left\{u_{j}\right\}
$$

for $j=1,2$.)

We say that a d-switching from $B \in \mathcal{C}_{d}$ specified by the (suitable) 8-tuple $T$ is legal if the resulting bipartite graph $B^{\prime}$ belongs to $\mathcal{C}_{d-1}$, and otherwise we say that the switching is illegal. Let $\operatorname{dist}_{\widehat{B}}(x, y)$ denote the length of the shortest path from $x$ to $y$ in a bipartite graph $\widehat{B}$.

Lemma 3.1. Suppose that $d \leqslant N_{2}$ is a positive integer and $B \in \mathcal{C}_{d}$. With notation as above, if the d-switching from $B$ specified by $T$ is illegal then at least one of the following conditions must hold: 


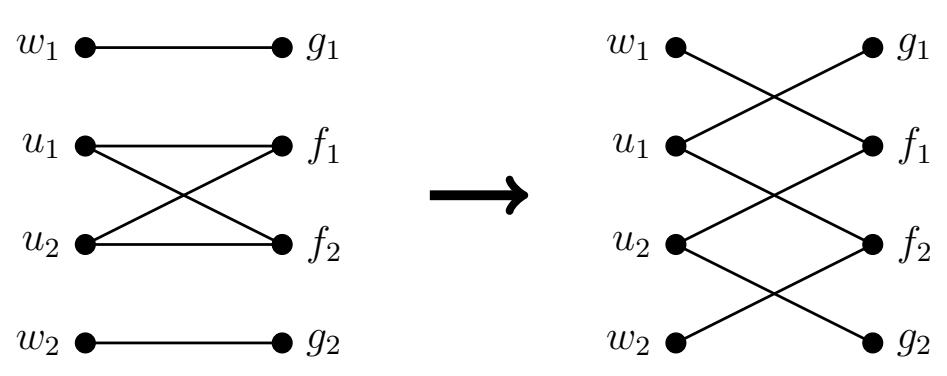

Figure 3: A d-switching

(I) At least one of $g_{1}$ or $g_{2}$ belongs to a 4-cycle in $B$.

(II) For some $j \in\{1,2\}$, either $\operatorname{dist}_{B}\left(u_{j}, g_{j}\right) \leqslant 3$ or $\operatorname{dist}_{B}\left(w_{j}, f_{j}\right) \leqslant 3$.

(III) $\operatorname{dist}_{B}\left(g_{1}, g_{2}\right)=2$.

Proof. Fix $B \in \mathcal{C}_{d}$ and let $T$ describe a d-switching from $B$ such that the resulting bipartite graph $B^{\prime}$ does not belong to $\mathcal{C}_{d-1}$. First, suppose that $B^{\prime} \in \mathcal{B}_{r}^{+}(\boldsymbol{k})$ but that $B^{\prime}$ contains at most $d-24$-cycles. Then the d-switching has destroyed more than one 4-cycle, which implies that (I) holds.

Next, suppose that there exists a 4-cycle in $B^{\prime}$ which contains an edge of $E\left(B^{\prime}\right) \backslash E(B)$. Call these new edges. Such a 4-cycle has been (accidently) created by the d-switching.

First suppose that $B^{\prime}$ contains a 4-cycle which involves precisely one new edge. If $B^{\prime}$ contains a 4 -cycle which involves the edge $u_{j} g_{j}$ (for some $j \in\{1,2\}$ ) but does not involve any other new edge then $\operatorname{dist}_{B}\left(u_{j}, g_{j}\right) \leqslant 3$, which implies that (II) holds. Similarly, if $B^{\prime}$ contains a 4-cycle which contains the edge $w_{j} f_{j}$ for some $j \in\{1,2\}$, but contains no other new edge, then $\operatorname{dist}_{B}\left(w_{j}, f_{j}\right) \leqslant 3$, which again implies that (II) holds. Now suppose that there are 4-cycles in $B^{\prime}$ which contain at least two new edges. If a 4-cycle in $B^{\prime}$ contains both $w_{1} f_{1}$ and $w_{2} f_{2}$ then $w_{1} f_{2} \in E(B)$, which implies that (II) holds. If a 4-cycle in $B^{\prime}$ contains both $u_{1} g_{1}$ and $u_{2} g_{2}$ then $u_{2} g_{1} \in E(B)$, so (II) holds. No 4-cycle in $B^{\prime}$ can contain both $u_{j} g_{j}$ and $w_{j} f_{j}$ for some $j \in\{1,2\}$, since the edge $u_{j} f_{j}$ is not present in $B^{\prime}$. Next, suppose that there is a 4-cycle in $B^{\prime}$ which contains both $u_{j} g_{j}$ and $w_{\ell} f_{\ell}$, where $\{j, \ell\}=\{1,2\}$. Then $w_{\ell} g_{j} \in E(B)$, so (III) holds.

The arguments given above cover the case that $B^{\prime} \in \mathcal{B}_{r}^{+}(\boldsymbol{k})$ but that $B^{\prime}$ has strictly more than $d-1$ 4-cycles, since the d-switching must have introduced at least one new 4-cycle. Furthermore, it follows from properties (i)-(v) that if $B^{\prime} \notin \mathcal{B}_{r}^{+}(\boldsymbol{k})$ then there must be a 4-cycle in $B^{\prime}$ which contains a new edge. (For example, if (i) fails for $B^{\prime}$ then there is a copy of $K_{3,2}$ involving at least one new edge, but then that new edge is contained in at least one 4-cycle in $B^{\prime}$.) Hence this case is also covered by the above arguments, completing the proof.

A reverse d-switching is the reverse of a d-switching. A reverse d-switching from a 
bipartite graph $B^{\prime} \in \mathcal{C}_{d-1}$ is described by a suitable 8-tuple $T$ of vertices such that

$$
u_{1} g_{1}, u_{2} g_{2}, u_{1} f_{2}, u_{2} f_{1}, w_{1} f_{1}, w_{2} f_{2}
$$

are all edges of $B^{\prime}$. The reverse d-switching produces the bipartite graph $B$ defined by (3.2). This operation is depicted in Figure 3 by following the arrow in reverse.

Given $B^{\prime} \in \mathcal{C}_{d-1}$, we say that a reverse d-switching from $B^{\prime}$ specified by the (suitable) 8-tuple $T$ is legal if the resulting bipartite graph $B$ belongs to $\mathcal{C}_{d}$, and otherwise we say that the switching is illegal.

The proof of the following is very similar to the proof of Lemma 3.1, but for completeness we give it in full.

Lemma 3.2. Suppose that $d \leqslant N_{2}$ is a positive integer and $B^{\prime} \in \mathcal{C}_{d-1}$. With notation as above, if the reverse switching from $B^{\prime}$ specified by $T$ is illegal then at least one of the following conditions must hold:

( $\left.\mathrm{I}^{\prime}\right)$ At least one of $u_{1}, u_{2}, f_{1}, f_{2}, g_{1}, g_{2}$ belongs to a 4 -cycle in $B^{\prime}$.

$\left(\mathrm{II}^{\prime}\right)$ For some $j \in\{1,2\}$, either $\operatorname{dist}_{B^{\prime}}\left(u_{j}, f_{j}\right) \leqslant 3$ or $\operatorname{dist}_{B^{\prime}}\left(w_{j}, g_{j}\right) \leqslant 3$.

Proof. Fix $B^{\prime} \in \mathcal{C}_{d-1}$ and let $T$ describe a reverse d-switching from $B^{\prime}$ such that the resulting bipartite graph $B$ does not belong to $\mathcal{C}_{d}$. First, suppose that $B \in \mathcal{B}_{r}^{+}(\boldsymbol{k})$ but that $B$ contains at most $d-14$-cycles. Then the reverse $d$-switching has destroyed at least one 4-cycle, so ( $\left.\mathrm{I}^{\prime}\right)$ holds.

Clearly any new 4-cycle in $B$ created by the reverse d-switching must contain at least one edge of $E(B) \backslash E\left(B^{\prime}\right)$. (Again, we call these new edges.) Of course, the reverse dswitching is designed to create a new 4-cycle involving the edges $u_{1} f_{1}, u_{1} f_{2}$, but here we are only interested in other 4-cycles which may "accidently" be created by the reverse d-switching.

If a 4 -cycle in $B$ contains precisely one new edge then (II') holds in $B^{\prime}$. Next suppose that a new 4-cycle in $B$ contains at least two new edges. If any new 4-cycle in $B$ contains both $w_{1} g_{1}$ and $w_{2} g_{2}$ then $w_{1} g_{2} \in E\left(B^{\prime}\right)$. This gives a 4 -cycle in $B^{\prime}$ involving $u_{2}$, and so (II') holds. No new 4-cycle in $B$ can contain both $u_{j} f_{j}$ and $w_{j} g_{j}$, since $u_{j} g_{j} \notin E(B)$, for any $j \in\{1,2\}$. Next, if $u_{j} f_{j}$ and $w_{\ell} g_{\ell}$ belong to a 4-cycle in $B$, where $\{j, \ell\}=\{1,2\}$, then $u_{j} g_{\ell} \in E\left(B^{\prime}\right)$ and $\left(\mathrm{II}^{\prime}\right)$ holds.

The above argument covers the possibility that $B^{\prime} \in \mathcal{B}_{r}^{+}(\boldsymbol{k})$ but that $B$ contains more than $d 4$-cycles. Now suppose that $B$ contains precisely $d 4$-cycles but $B \notin \mathcal{B}_{r}^{+}(\boldsymbol{k})$. Note that property (v) holds, by our assumption on $d$. If property (i) or (ii) fails for $B$ then at least one additional 4-cycle has been created by the reverse d-switching, which was covered by the above argument. If property (iii) fails for $B$ then either $f_{1}$ or $f_{2}$ must belong to a 4-cycle in $B^{\prime}$, while if property (iv) fails for $B$ then either $u_{1}$ or $u_{2}$ must belong to a 4-cycle in $B^{\prime}$. Thus ( $\left.\mathrm{I}^{\prime}\right)$ holds in both cases, completing the proof.

We will analyse d-switchings to obtain an asymptotic expression for $\left|\mathcal{C}_{d}\right| /\left|\mathcal{C}_{d-1}\right|$, and then combine these to find an expression for $\left|\mathcal{L}_{r}(\boldsymbol{k})\right|=\left|\mathcal{C}_{0}\right| /(M / r)$ !, which is the quantity of interest. First we analyse one d-switching. 
Lemma 3.3. Assume that the conditions of Theorem 1.1 hold. Let $d^{\prime}$ be the first value of $d \leqslant N_{2}$ such that $\mathcal{C}_{d}=\varnothing$, or $d^{\prime}=N_{2}+1$ if no such value exists. If $d \in\left\{1, \ldots, d^{\prime}-1\right\}$ then

$$
\left|\mathcal{C}_{d}\right|=\left|\mathcal{C}_{d-1}\right| \frac{(r-1)^{2} M_{2}^{2}}{4 d M^{2}}\left(1+O\left(\frac{d k_{\max }\left(k_{\max }+r\right)+r^{2} k_{\max }^{3}}{M_{2}}\right)\right) .
$$

Proof. Fix $d \in\left\{1, \ldots, d^{\prime}-1\right\}$ and let $B \in \mathcal{C}_{d}$ be given. Let $\mathcal{S}$ be the set of all suitable 8-tuples $T$ such that

- $B$ contains a 4 -cycle on $\left\{u_{1}, u_{2}, f_{1}, f_{2}\right\}$,

- the edges $w_{1} g_{1}, w_{2} g_{2}$ belong to $B$, and

- no 4-cycle in $B$ contains $g_{1}$ or $g_{2}$.

Then $\mathcal{S}$ contains every 8-tuple which defines a legal d-switching from $B$, so $|\mathcal{S}|$ is an upper bound for the number of legal d-switchings from $B$. There are precisely $d 4$-cycles, and 4 ways to order the vertices $\left(u_{1}, u_{2}, f_{1}, f_{2}\right)$. For an upper bound, there are at most $M^{2}$ ways to choose the edges $w_{1} g_{1}, w_{2} g_{2}$ in order, giving $|\mathcal{S}| \leqslant 4 d M^{2}$. To give a lower bound, we must ensure that all vertices are distinct and that $g_{1}$ and $g_{2}$ are not contained in any 4-cycle. Given $\left(u_{1}, u_{2}, f_{1}, f_{2}\right)$, there are at least

$$
\left(M-\left(2 r d+2 k_{\max }\right)\right)\left(M-\left((2 d+1) r+3 k_{\max }\right)\right)
$$

good choices for $\left(w_{1}, w_{2}, g_{1}, g_{2}\right)$. Hence

$$
|\mathcal{S}|=4 d M^{2}\left(1+O\left(\frac{r d+k_{\max }}{M}\right)\right) \text {. }
$$

We now find obtain an upper bound for the number of 8-tuples in $\mathcal{S}$ which give rise to illegal d-switchings from $B$, and subtract this value from $|\mathcal{S}|$. By Lemma 3.1 it suffices to find an upper bound for the number of 8-tuples in $\mathcal{S}$ which satisfy Condition (II) or Condition (III). Observe that no 8-tuple in $\mathcal{S}$ satisfies Condition (I), by definition of $\mathcal{S}$. For Condition (II), there are $O\left(d r k_{\max } M\right)$ 8-tuples in $\mathcal{S}$ such that an edge exists in $B$ from $u_{1}$ to $g_{j}$ or from $w_{j}$ to $f_{j}$, for some $j \in\{1,2\}$. Similarly, there are $O\left(d r^{2} k_{\max }^{2} M\right)$ 8-tuples for which $\operatorname{dist}_{B}\left(u_{1}, g_{j}\right)=3 \operatorname{or}_{\operatorname{dist}_{B}}\left(w_{j}, f_{j}\right)=3$, for some $j \in\{1,2\}$. Hence Condition (II) fails for $O\left(d r^{2} k_{\max }^{2} M\right)$ 8-tuples in $\mathcal{S}$.

Similarly, there are $O\left(d r^{2} k_{\max } M\right)$ 8-tuples in $\mathcal{S}$ which satisfy Condition (III). Combining these contributions, it follows that there are

$$
4 d M^{2}\left(1+O\left(\frac{r d+r^{2} k_{\max }^{2}}{M}\right)\right)
$$

suitable 8-tuples which give a legal d-switching from $B$.

Next, suppose that $B^{\prime} \in \mathcal{C}_{d-1}$ (and note that $\mathcal{C}_{d-1}$ is nonempty, by definition of $d^{\prime}$ ). Let $\mathcal{S}^{\prime}$ be the set of all suitable 8-tuples such that 
- $u_{1} g_{1}, u_{2} g_{2}, u_{1} f_{2}, u_{2} f_{1}, w_{1} f_{1}, w_{2} f_{2}$ are all edges of $B^{\prime}$, and

- no 4-cycle in $B^{\prime}$ contains a vertex from $\left\{u_{1}, u_{2}, f_{1}, f_{2}, g_{1}, g_{2}\right\}$.

Again, $\mathcal{S}^{\prime}$ contains every 8-tuple which describes a legal reverse d-switching from $B^{\prime}$. Hence there are at most $\left|\mathcal{S}^{\prime}\right|$ legal reverse d-switchings from $B^{\prime}$. There are at most $M_{2}$ ways to choose $\left(u_{1}, f_{2}, g_{1}\right)$ and at most $M_{2}$ ways to choose $\left(u_{2}, f_{1}, g_{2}\right)$, and then at most $(r-1)^{2}$ ways to choose $\left(w_{1}, w_{2}\right)$. Therefore $\left|\mathcal{S}^{\prime}\right| \leqslant(r-1)^{2} M_{2}^{2}$.

For a lower bound, we must ensure that all vertices are distinct and that we avoid choosing $u_{1}, u_{2}, f_{1}, f_{2}, g_{1}, g_{2}$ from a 4 -cycle. We can choose $\left(u_{1}, f_{2}, g_{1}\right)$, avoiding vertices contained in 4-cycles, in at least $M_{2}-2(d-1) k_{\max }\left(k_{\max }+2 r\right)$ ways. There are still precisely $(r-1)$ choices for $w_{2}$ from among all neighbours of $f_{2}$ other than $u_{1}$. Next, there are at least

$$
M_{2}-2(d-1) k_{\max }\left(k_{\max }+2 r\right)-3 k_{\max }^{2}-4 r k_{\max }-2 r k_{\max }^{2}
$$

ways to choose $\left(u_{2}, f_{1}, g_{2}\right)$ avoiding vertices contained in 4-cycles and avoiding those vertices already chosen, such that $f_{1}$ is not a neighbour of $u_{1}$ or $w_{2}$ in $B^{\prime}$. This choice of $f_{1}$ ensures that all $r-1$ neighbours of $f_{1}$ other than $u_{2}$ are also distinct from $\left\{u_{1}, w_{2}\right\}$, so there are still $r-1$ choices for $w_{2}$. It follows that

$$
\left|\mathcal{S}^{\prime}\right|=(r-1)^{2} M_{2}^{2}\left(1+O\left(\frac{d k_{\max }\left(k_{\max }+r\right)+r k_{\max }^{2}}{M_{2}}\right)\right) .
$$

Now we calculate an upper bound for the number of 8-tuples in $\mathcal{S}^{\prime}$ which give an illegal reverse d-switching from $B^{\prime}$. By Lemma 3.2, it suffices to find an upper bound for the number of 8-tuples in $\mathcal{S}^{\prime}$ which satisfy Condition $\left(\mathrm{II}^{\prime}\right)$. (Note that no element of $\mathcal{S}^{\prime}$ can satisfy Condition $\left(\mathrm{I}^{\prime}\right)$, by definition of $\mathcal{S}^{\prime}$.) There are $O\left(r^{3} k_{\max }^{2} M_{2}\right)$ elements of $\mathcal{S}^{\prime}$ such that there is an edge from $w_{j}$ to $g_{j}$ or an edge from $u_{j}$ to $f_{j}$, for some $j \in\{1,2\}$. Similarly, the number of 8-tuples in $\mathcal{S}^{\prime}$ with $\operatorname{dist}_{B^{\prime}}\left(u_{j}, f_{j}\right)=3$ or with $\operatorname{dist}_{B^{\prime}}\left(w_{j}, g_{j}\right)=3$ for some $j \in\{1,2\}$ is $O\left(r^{4} k_{\max }^{3} M_{2}\right)$. Hence Condition (II') fails for $O\left(r^{4} k_{\max }^{3} M_{2}\right)$ 8-tuples, which (together with the upper bound on $\left|\mathcal{S}^{\prime}\right|$ ) implies that the number of legal reverse d-switchings from $B^{\prime}$ is

$$
(r-1)^{2} M_{2}^{2}\left(1+O\left(\frac{d k_{\max }\left(k_{\max }+r\right)+r^{2} k_{\max }^{3}}{M_{2}}\right)\right) .
$$

Comparing the error terms from (3.3) and (3.4), we see that the error term from the reverse d-switchings is largest, since $1 / M \leqslant k_{\max } / M_{2}$. Taking the ratio of (3.3) and (3.4) completes the proof.

We can now prove our main result. The proof is similar to those in related enumeration results such as [9]. We present the proof in full in order to demonstrate how the factors of $r$ arise in the error bounds (since previous results only dealt with $r=2$, or assumed that $r$ was constant). The following summation lemma from [10] will be needed. (The statement has been adapted slightly from that given in [10], without affecting the proof given there.) 
Lemma 3.4 ([10, Corollary 4.5]). Let $N \geqslant 2$ be an integer and, for $1 \leqslant i \leqslant N$, let real numbers $A(i), C(i)$ be given such that $A(i) \geqslant 0$ and $A(i)-(i-1) C(i) \geqslant 0$. Define $A_{1}=\min _{i=1, \ldots, N} A(i), A_{2}=\max _{i=1, \ldots, N} A(i), C_{1}=\min _{i=1, \ldots, N} C(i)$ and $C_{2}=$ $\max _{i=1, \ldots, N} C(i)$. Suppose that there exists a real number $\hat{c}$ with $0<\hat{c}<\frac{1}{3}$ such that $\max \left\{A_{2} / N,\left|C_{1}\right|,\left|C_{2}\right|\right\} \leqslant \hat{c}$. Define $n_{0}, \ldots, n_{N}$ by $n_{0}=1$ and

$$
n_{i}=\frac{1}{i}(A(i)-(i-1) C(i)) n_{i-1}
$$

for $1 \leqslant i \leqslant N$. Then

$$
\Sigma_{1} \leqslant \sum_{i=0}^{N} n_{i} \leqslant \Sigma_{2}
$$

where

$$
\begin{aligned}
& \Sigma_{1}=\exp \left(A_{1}-\frac{1}{2} A_{1} C_{2}\right)-(2 e \hat{c})^{N}, \\
& \Sigma_{2}=\exp \left(A_{2}-\frac{1}{2} A_{2} C_{1}+\frac{1}{2} A_{2} C_{1}^{2}\right)+(2 e \hat{c})^{N} .
\end{aligned}
$$

Proof of Theorem 1.1. First we prove that

$$
\sum_{d=0}^{N_{2}}\left|\mathcal{C}_{d}\right|=\left|\mathcal{C}_{0}\right| \exp \left(\frac{(r-1)^{2} M_{2}^{2}}{4 M^{2}}+O\left(\frac{r^{4} k_{\max }^{4}\left(k_{\max }+r\right)}{M}\right)\right) .
$$

Let $d^{\prime}$ be the first value of $d \leqslant N_{2}$ for which $\mathcal{C}_{d}=\varnothing$, or $d=N_{2}+1$ if no such value of $d$ exists. We saw in Lemma 3.3 that any $B \in \mathcal{C}_{d}$ can be converted to some $B^{\prime} \in \mathcal{C}_{d-1}$ using a d-switching. Hence $\mathcal{C}_{d}=\varnothing$ for $d^{\prime} \leqslant d \leqslant N_{2}$. In particular, (3.5) holds if $\mathcal{C}_{0}=\varnothing$, so we assume that $d^{\prime} \geqslant 1$.

By Lemma 3.3, there is some uniformly bounded function $\alpha_{d}$ such that

$$
\frac{\left|\mathcal{C}_{d}\right|}{\left|\mathcal{C}_{0}\right|}=\frac{1}{d} \frac{\left|\mathcal{C}_{d-1}\right|}{\left|\mathcal{C}_{0}\right|}(A(d)-(d-1) C(d))
$$

for $1 \leqslant d \leqslant N_{2}$, where

$$
A(d)=\frac{(r-1)^{2} M_{2}^{2}-\alpha_{d} r^{4} k_{\max }^{3} M_{2}}{4 M^{2}}, \quad C(d)=\frac{\alpha_{d} r^{2} k_{\max }\left(k_{\max }+r\right) M_{2}}{4 M^{2}}
$$

for $1 \leqslant d<d^{\prime}$, and $A(d)=C(d)=0$ for $d^{\prime} \leqslant d \leqslant N_{2}$.

We wish to apply Lemma 3.4. It is clear that $A(d)-(d-1) C(d) \geqslant 0$, from (3.6) if $1 \leqslant d<d^{\prime}$ or by definition, if $d^{\prime} \leqslant d \leqslant N_{2}$. If $\alpha_{d} \geqslant 0$ then $A(d) \geqslant A(d)-(d-1) C(d) \geqslant 0$ by (3.6), while if $\alpha_{d}<0$ then $A(d)$ is nonnegative by definition. Now define $A_{1}, A_{2}, C_{1}, C_{2}$ by taking the minimum and maximum of $A(d)$ and $C(d)$ over $1 \leqslant d \leqslant N_{2}$. Let $A \in\left[A_{1}, A_{2}\right]$ and $C \in\left[C_{1}, C_{2}\right]$ and set $\hat{c}=\frac{1}{20}$. Since $A=(r-1)^{2} M_{2}^{2} / 4 M^{2}+o(1)$ and $C=o(1)$, we 
have that $\max \left\{A / N_{2},|C|\right\} \leqslant \hat{c}$ for $M$ sufficiently large, by the definition of $N_{2}$. Hence Lemma 3.4 applies and gives an upper bound

$$
\sum_{d=0}^{N_{2}} \frac{\left|\mathcal{C}_{d}\right|}{\left|\mathcal{C}_{0}\right|} \leqslant \exp \left(\frac{(r-1)^{2} M_{2}^{2}}{4 M^{2}}+O\left(\frac{r^{4} k_{\max }^{4}\left(k_{\max }+r\right)}{M}\right)\right)+O\left((e / 10)^{N_{2}}\right) .
$$

Since $(e / 10)^{N_{2}} \leqslant(e / 10)^{3 \log M} \leqslant M^{-1}$, this gives

$$
\sum_{d=0}^{N_{2}} \frac{\left|\mathcal{C}_{d}\right|}{\left|\mathcal{C}_{0}\right|} \leqslant \exp \left(\frac{(r-1)^{2} M_{2}^{2}}{4 M^{2}}+O\left(\frac{r^{4} k_{\max }^{4}\left(k_{\max }+r\right)}{M}\right)\right) .
$$

In the case that $d^{\prime}=N_{2}+1$, the lower bound given by Lemma 3.4 is the same within the stated error term, which establishes (3.5) in this case.

This leaves the case that $1 \leqslant d^{\prime} \leqslant N_{2}$. Considering the analysis of the reverse switchings from Lemma 3.3, this case can only arise if

$$
M_{2}=O\left(d^{\prime} k_{\max }\left(k_{\max }+r\right)+r^{2} k_{\max }^{3}\right)=O\left(k_{\max }\left(k_{\max }+r\right)\left(r^{2} k_{\max }^{2}+\log M\right)\right) .
$$

But then

$$
\frac{(r-1)^{2} M_{2}^{2}}{4 M^{2}}=O\left(\frac{r^{2} k_{\max }^{2}\left(k_{\max }+r\right)^{2}\left(r^{2} k_{\max }^{2}+\log M\right)^{2}}{M^{2}}\right)=O\left(\frac{r^{4} k_{\max }^{4}}{M}\right)
$$

so the trivial lower bound of 1 matches the upper bound within the error term. Hence (3.5) also holds when $1 \leqslant d^{\prime} \leqslant N_{2}$.

Therefore (3.5) holds in both cases. Combining (2.1), Theorem 2.2 and (3.1) gives

$$
\begin{aligned}
& \left|\mathcal{L}_{r}(\boldsymbol{k})\right| \\
& =\frac{\left|\mathcal{C}_{0}\right|}{(M / r) !} \\
& =\frac{\left|\mathcal{B}_{r}^{+}(\boldsymbol{k})\right|}{(M / r) !} \exp \left(-\frac{(r-1)^{2} M_{2}^{2}}{4 M^{2}}+O\left(\frac{r^{4} k_{\max }^{4}\left(k_{\max }+r\right)}{M}\right)\right) \\
& =\frac{M !}{(M / r) !(r !)^{M / r} \prod_{j=1}^{n} k_{j} !} \exp \left(-\frac{(r-1) M_{2}}{2 M}-\frac{(r-1)^{2} M_{2}^{2}}{4 M^{2}}+O\left(\frac{r^{4} k_{\max }^{4}\left(k_{\max }+r\right)}{M}\right)\right),
\end{aligned}
$$

completing the proof.

As a corollary of Theorem 2.2 and (3.5) we obtain the following result regarding the girth of bipartite graphs.

Corollary 3.5. Under the conditions of Theorem 1.1, the probability that a randomly chosen element of $\mathcal{B}_{r}(\boldsymbol{k})$ has no 4-cycle, and hence has girth at least 6 , is

$$
\frac{\left|\mathcal{C}_{0}\right|}{\left|\mathcal{B}_{r}(\boldsymbol{k})\right|}=\exp \left(-\frac{(r-1)^{2} M_{2}^{2}}{4 M^{2}}+O\left(\frac{r^{4} k_{\max }^{4}\left(k_{\max }+r\right)}{M}\right)\right) \text {. }
$$


McKay, Wormald and Wysocka [18, Corollary 3] proved the following: if $(d-1)^{7}=o(n)$ as $n \rightarrow \infty$ along the positive even integers then that the probability that a random $d$ regular bipartite graph on $n$ vertices has girth greater than $g$ is

$$
\exp \left(-\frac{(d-1)^{4}}{4}+o(1)\right)
$$

(The conclusion was known much earlier for constant $d$; see [20].) Corollary 3.5 can be seen as a generalisation of the $g=4$ case of [18, Corollary 3] to bipartite graphs which are irregular on one side of the vertex bipartition, and are sufficiently sparse. When the bipartite graph is $d$-regular (with $k_{\max }=r=d$ ), the condition of Corollary 3.5 becomes $d^{8}=o(n)$, which is slightly more restrictive than that of [18].

\section{Acknowledgements}

We are very grateful to Brendan McKay for suggesting that use of the incidence matrix of the hypergraph would simplify the calculations. We thank the referee for their helpful comments.

\section{References}

[1] C. Berge, Hypergraphs: Combinatorics of Finite Sets, Elsevier, Amsterdam, 1989.

[2] V. Blinovsky and C. Greenhill, Asymptotic enumeration of sparse uniform hypergraphs with given degrees, European Journal of Combinatorics 51 (2016), 287-296.

[3] A. Chattopadhyay and B. A. Reed, Properly 2-colouring linear hypergraphs, in Approximation, Randomization, And Combinatorial Optimization. Algorithms and Techniques, Lecture Notes in Computer Science 4627 (2007), pp. 395-408.

[4] C. Cooper, A. Frieze, M. Molloy and B. Reed, Perfect matchings in random $r$-regular, s-uniform hypergraphs, Combinatorics, Probability and Computing 5 (1996), 1-14.

[5] A. Dudek, A. Frieze, A. Ruciński and M. Šileikis, Approximate counting of regular hypergraphs, Information Processing Letters 113 (2013), 785-788.

[6] E. Estrada, J. A. Rodríguez-Velázquez, Subgraph centrality and clustering in complex hyper-networks, Physica A: Statistical Mechanics and its Applications 364 (2006), 581-594.

[7] A. Frieze and P. Melsted, Randomly coloring simple hypergraphs, Information Processing Letters 111 (2011), 848-853.

[8] G. Ghoshal, V. Zlatić, G. Caldarelli and M. E. J. Newman, Random hypergraphs and their applications, Physical Review E 79 (2009), 066118.

[9] C. Greenhill and B. D. McKay, Asymptotic enumeration of sparse multigraphs with given degrees, SIAM Journal on Discrete Mathematics 27 (2013), 2064-2089. 
[10] C. Greenhill, B. D. McKay and X. Wang, Asymptotic enumeration of sparse 0-1 matrices with irregular row and column sums, Journal of Combinatorial Theory (Series A) $\mathbf{1 1 3}$ (2006), 291-324.

[11] S. Klamt, U.-U. Haus and F. Theis, Hypergraphs and cellular networks, PLoS Comput. Biol. 5 (2009) e31000385.

[12] A.V. Kostochka and M. Kumbhat, Coloring uniform hypergraphs with few edges, Random Structure and Algorithms 35 (2009), 348-368.

[13] G. Kuperberg, S. Lovett and R. Peled, Probabilistic existence of regular combinatorial structures, Preprint, 2013. arXiv:1302.4295 (Extended abstract appeared in the Proceedings of the 44th Annual ACM Symposium on the Theory of Computing, New York, 2012, pp. 1091-1106.)

[14] B. D. McKay, Subgraphs of random graphs with specified degrees, Congressum Numerantium 33 (1981), 213-223.

[15] B. D. McKay, Subgraphs of dense random graphs with specified degrees, Combin. Probab. Comput., 20 (2011) 413-433.

[16] B. D. McKay and N. C. Wormald, Asymptotic enumeration by degree sequence of graphs of high degree, European J. Combin., 11 (1990) 565-580.

[17] B. D. McKay and N. C. Wormald, Asymptotic enumeration by degree sequence of graphs with degrees $o\left(n^{1 / 2}\right)$, Combinatorica 11 (1991), 369-383.

[18] B. D. McKay, N. C. Wormald and B. Wysocka, Short cycles in random regular graphs, Electronic Journal of Combinatorics 11(1) (2004), \#R66.

[19] R. N. Naik, S. B. Rao, S. S. Shrikhande and N. M. Singhi, Intersection graphs of $k$ uniform linear hypergraphs, European Journal of Combinatorics 3 (1982), 159-172.

[20] N. C. Wormald, Some Problems in the Enumeration of Labelled Graphs, Ph.D. thesis, University of Newcastle, 1978. 